\title{
Excitons and Trions in Modulation Doped Structures in High Magnetic Fields
}

\author{
V. Kochereshko, D. Andronikov, A. Platonov
}

A.F. Ioffe Physico-Technical Institute RAS, 194021 St. Petersburg, Russia

S. Crooker, T. Barrick

National High Magnetic Field Laboratory

Los Alamos, New Mexico 87545, USA

\section{G. KARCZEWSKI}

Institute of Physics, Polish Academy of Sciences

al. Lotników 32/46, 02-668 Warsaw, Poland

\section{AND P. TRONC}

Laboratoire d'Optique, Ecole Supérieure de Physique et Chimie Industrielles

10 rue Vauquelin, 75005 Paris, France

Photoluminescence spectra of modulation-doped CdTe/CdMgTe quantum well structures containing two-dimensional electron gases of low, moderate and high electron concentrations were studied in high magnetic fields up to $45 \mathrm{~T}$. The recombination line of triplet trion state was found in the spectra. A model calculation of photoluminescence spectra in magnetic fields, which takes into account singlet and triplet trion states, was carried out. It was shown that the dark triplet becomes observable in photoluminescence spectra because it becomes the only recombination channel when the formation of the singlet trion state is suppressed by magnetic fields.

PACS numbers: 78.55.Et, 71.35.--y, 78.20.Ls, 78.40.Fy

\section{Introduction}

Charged exciton-electron complexes (trions) are bound states of three particles. They consist of two electrons and one hole in the case of negatively charged excitons, and two holes and one electron in the case of positively charged exci- 
tons [1]. The ground state of a trion in zero magnetic fields is a singlet, i.e. the two electrons (two holes) have opposite spin alignment. The triplet state of a trion, in which these charged particles have the same spin alignment, is not a bound state in the absence of magnetic fields. Observations of the triplet states were reported in a number of publications devoted to studies of quantum wells based on ZnSe [2] and GaAs [3, 4]. However, as the authors of the articles mentioned above note, the question "why are the triplet states of the trion observed?" remains unresolved. Theoretical calculations show that optically dark and optically active triplet states of trions can exist with the dark state being the lowest energy triplet state [5].

This puts into question whether the observed lines were correctly identified. In [6] the observation of the triplet state was explained by the singlet-triplet mixing when the trion interacts with the additional electrons. In [7] the appearance of the oscillator strength of the triplet trion was explained by the anticrossing of the triplet state with the singlet state of the trion. All the published data concerning the triplet states of the trions were obtained on the structures with ZnSe- or GaAs-based quantum wells (QWs).

In the present work the triplet trion states were studied in CdTe-based quantum well structures. In these materials the sign of the $g$-factors of the electron and hole are opposite to each other. Thus the energy structure of the exciton and trion states under magnetic field in these materials is different from that in ZnSe. This allows us to check the idea that the anticrossing of the singlet and triplet states affects the oscillator strength of the triplet trion and to identify the spin structure of the observed triplet trion state.

\section{Background}

The point symmetry of wave functions in QWs is described by the $D_{2 d}$ point group. When applying a magnetic field parallel to the growth axis the translational symmetry of the system in the layer plane is removed and its symmetry is reduced to the $S_{4}$ point group.

Carriers and trions are fermions. In magnetic fields their symmetry is described by the $\Gamma_{5-8}$ double-valued irreducible representations (irreps) of the $S_{4}$ group. Any single- or double-valued irrep of the $S_{4}$ point group is unidimensional. The $\Gamma_{5}$ and $\Gamma_{7}\left(\Gamma_{6}\right.$ and $\left.\Gamma_{8}\right)$ irreps are complex conjugate (coreps). Within each pair of coreps, the two irreps correspond to opposite spin values. The vector representation in the $S_{4}$ group is $\Gamma_{\mathrm{v}}=\Gamma_{1}(z)+\Gamma_{3}(x-\mathrm{i} y)+\Gamma_{4}(x+\mathrm{i} y)$.

Excitons are bosons. Their symmetry is described by the $\Gamma_{1-4}$ single-valued irreps. The $\Gamma_{3}$ and $\Gamma_{4}$ excitons are bright and recombine in $\left(\sigma^{+}\right)$and $\left(\sigma^{-}\right)$polarization, respectively. The $\Gamma_{2}$ excitons are dark.

The symmetry of a trion made of a h hole, an $\mathrm{e}_{1}$ electron, and an $\mathrm{e}_{2}$ electron is included in the $\Gamma_{\mathrm{h}} \times \Gamma_{\mathrm{e} 1} \times \Gamma_{\mathrm{e} 2} \times \Gamma_{\mathrm{env}}$ direct product, where $\Gamma_{\mathrm{h}}, \Gamma_{\mathrm{e} 1}, \Gamma_{\mathrm{e} 2}$, and $\Gamma_{\text {env }}$ are the irreps describing the symmetries of the h hole, $\mathrm{e}_{1}$ electron, $\mathrm{e}_{2}$ electron, 
and envelope-functions corresponding to the relative motions of the three particles within the trion, respectively. The $\Gamma_{\text {env }}$ irrep is certainly $\Gamma_{1}$ for the ground state of a trion with any given symmetry. It should be the vector representation for the first excited states. That corresponds to the $s$ and $p$ states of the orbital momentum in the hydrogen-like approximation.

Similar to the negatively charged hydrogen ion $\mathrm{H}^{-}$, the trion $\mathrm{X}^{-}$also has two independent sets of states - triplet and singlet. In the singlet state two electrons in a trion have total spin $S_{\mathrm{e}}=0$. The orbital wave function of this state is symmetrical under permutation of electrons. One can consider that each of the electrons is in the $1 S$ state of orbital motion but each with its own Bohr radius. The orbital momentum of such a state is $L=0$. Optical transitions into such states are allowed.

In the triplet states two electrons have total spin $S_{\mathrm{e}}= \pm 1$. The orbital wave function of this state is antisymmetrical to permutation of electrons. In such a state the wave function is different from zero only either if the full orbital momentum is different from zero $(L \neq 0)$ or if the electrons are on different orbitals e.g. $1 S$ and $2 S$.

It is known [5] that the lowest energy trion triplet state which usually becomes observable in experiments has an orbital momentum projection on the magnetic field $L_{z}=-1$ and thus is optically forbidden. It can become optically active only due to spin-orbit interaction or due to the additional orbital momentum of the hole. The state with the projection of the electron orbital momentum $L_{z}=0$ is "connected" to the second Landau level i.e. it is energetically higher than the considered energy range by the magnitude of the cyclotron energy of the electron.

\section{Experiment}

We studied modulation-doped $\mathrm{CdTe} /\left(\mathrm{Cd}_{0.7}, \mathrm{Mg}_{0.3}\right) \mathrm{Te} \mathrm{QW}$ structures that contained a two-dimensional electron gas (2DEG) with electron density that varied from $n_{\mathrm{e}}<10^{10}$ up to $10^{12} \mathrm{~cm}^{-2}$. These structures contained a $100 \AA$ single quantum well (SQW) grown on GaAs (100) substrates, and were $\delta$-doped in the barriers at $100 \AA$ distance from the QW. A special design of the structures made it possible to control the electron concentration while keeping all other QW parameters (QW width, barrier height, background impurity concentration, etc.) constant with high accuracy.

Polarized photoluminescence from these samples was measured with magnetic fields applied in the Faraday configuration. Experiments were performed in a capacitor-driven $50 \mathrm{~T}$ mid-pulse magnet (400 ms pulse duration). A complete set of field-dependent photoluminescence (PL) spectra excited by a $\mathrm{He}-\mathrm{Cd}$ laser at $1.6 \mathrm{~K}$ temperature was collected during each magnet pulse. Optical fibers were used for optical excitation of the sample, and the emitted light was detected in both circular polarizations, allowing identification of the spin components of excitons and trions. 
Figure 1 shows a set of photoluminescence (PL) spectra taken in magnetic fields from 0 to $45 \mathrm{~T}$ in left and right circular polarization $\left(\sigma^{-}\right.$and $\left.\sigma^{+}\right)$in a structure with a single QW and electron concentration $3 \times 10^{10} \mathrm{~cm}^{-2}$. In the PL spectra in low magnetic fields a bright PL line $\left(T_{\mathrm{S}}\right)$ is observed at the energy of $\approx 1.614 \mathrm{eV}$. This line is attributed to the singlet state of a charged exciton complex - the trion $[1,8]$. With increasing magnetic field, a PL line of the neutral exciton appears in the spectra in $\sigma^{-}$polarization. It is shifted $3 \mathrm{meV}$ to the high-energy side from the trion line. The absence of the exciton line in the PL spectra in low magnetic field is connected with the rapid binding of the photocreated exciton with an extra electron (much faster than exciton radiative recombination) [9]. At intermediate magnetic fields a new line ( $T_{\mathrm{t}}$ in Fig. 1$)$ appears in the PL spectra in the energy range between the exciton and trion lines.
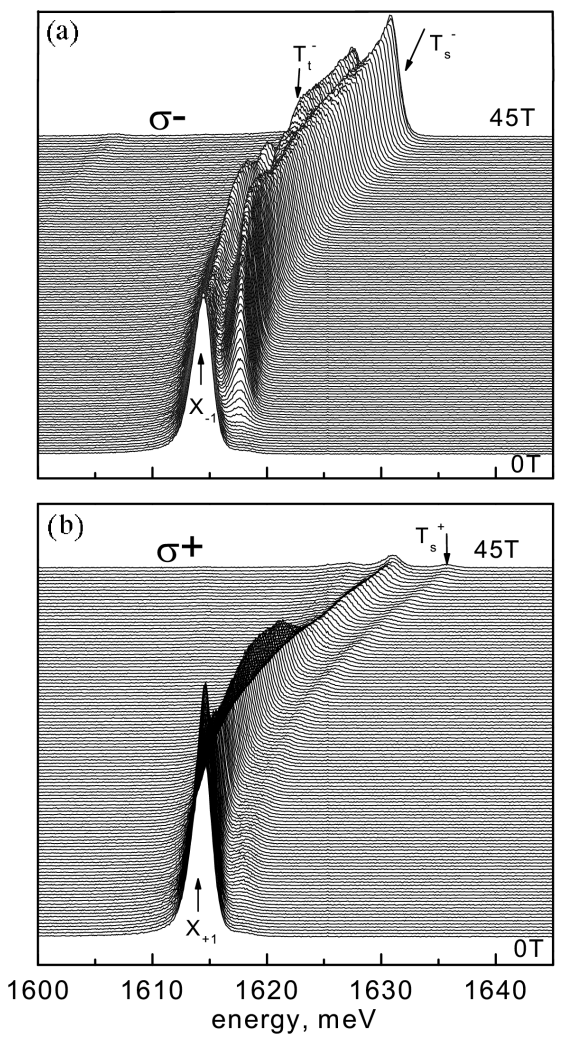

Fig. 1. Set of photoluminescence spectra for $\mathrm{CdTe} / \mathrm{Cd}_{0.7} \mathrm{Mg}_{0.3} \mathrm{Te} \mathrm{SQW}$ structure with electron density $3 \times 10^{10} \mathrm{~cm}^{-2}$ in magnetic fields from 0 to $45 \mathrm{~T}$ at $1.6 \mathrm{~K}$ in (a) left $\sigma^{-}$and (b) right $\sigma^{+}$circular polarizations. Arrows $\left(X_{-1}, T_{-}^{\mathrm{s}}\right)\left(X_{+1}, T_{+}^{\mathrm{s}}\right)$ mark the exciton and singlet trion lines in $\sigma^{-}$and $\sigma^{+}$polarizations. $T_{\mathrm{t}}$ is the triplet trion line. 


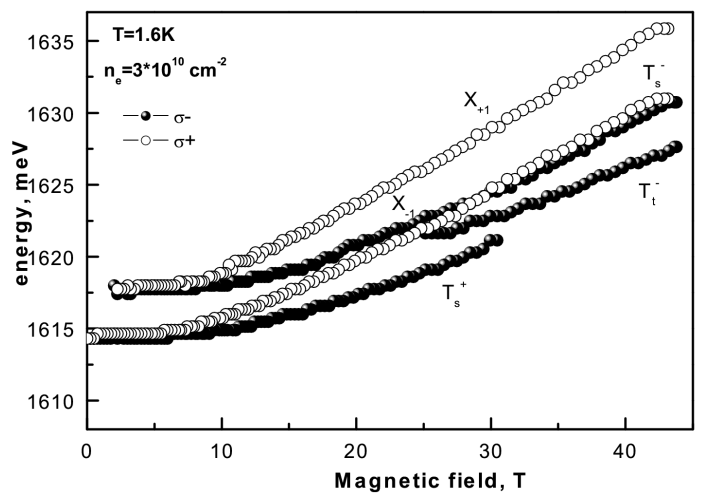

Fig. 2. Magnetic field dependences of the energy positions of the $X, T_{\mathrm{s}}$, and $T_{\mathrm{t}} \mathrm{PL}$ lines observed in $\sigma^{+}$and $\sigma^{-}$polarizations. Labels $\left(X_{-1}, T_{-}^{\mathrm{s}}\right)\left(X_{+1}, T_{+}^{\mathrm{s}}\right)$ mark the exciton and singlet trion lines in $\sigma^{-}$and $\sigma^{+}$polarizations. $T_{\mathrm{t}}$ is the triplet trion line.

Figure 2 shows the dependences of the energy positions of the maxima of all the lines in the PL spectra. The exciton $X$ and trion $T_{\mathrm{s}}$ emission lines experience a diamagnetic shift towards high energy of the spectrum that is approximately the same for all the lines. Besides the diamagnetic shift, a Zeeman splitting of these lines is observed. The magnitude of this splitting is the same for the exciton $X$ and the singlet trion $T_{\mathrm{s}}$. In magnetic fields higher than $25 \mathrm{~T}$, near the crossing between the upper Zeeman component of the trion and the lower Zeeman component of the exciton, a new line $\left(T_{\mathrm{t}}\right)$ appears. We identify this line as the triplet trion state. This line shifts towards lower energies from the exciton PL line and also experiences a diamagnetic shift. Theoretical calculations show that in the absence of magnetic field the triplet trion state is not bound, and becomes bound only in high enough magnetic fields.

Figure 3 shows the dependences of the intensity of all the PL lines on the magnetic field. In low magnetic fields the intensity of the trion line is much larger than that of the exciton, which is due to the rapid rate of trion formation. With increasing magnetic field the intensity of the trion line $T_{\mathrm{s}}$ decreases both in $\sigma^{+}$ and $\sigma^{-}$circular polarizations, while the intensity of the exciton line $X$ increases in $\sigma^{-}$polarization and remains constant in $\sigma^{+}$polarization. These peculiarities of the behavior of the polarized photoluminescence of the excitons and trions in QWs, containing a 2DEG are analyzed in detail in [10], where it is shown that they are linked to the fast spin-dependent processes of the trion formation.

It is noteworthy that the recombination line of the triplet state of the trion $T_{\mathrm{t}}$ is observed only in the $\sigma^{-}$circular polarization and the intensity of this line does not depend on the magnetic field in the whole range of fields where it is observed ( 25 to $45 \mathrm{~T})$.

The energy splitting of the Zeeman components of the exciton PL line allows one to determine the $g$-factor of the optically active state of the exciton $g_{\text {exc }}=$ 


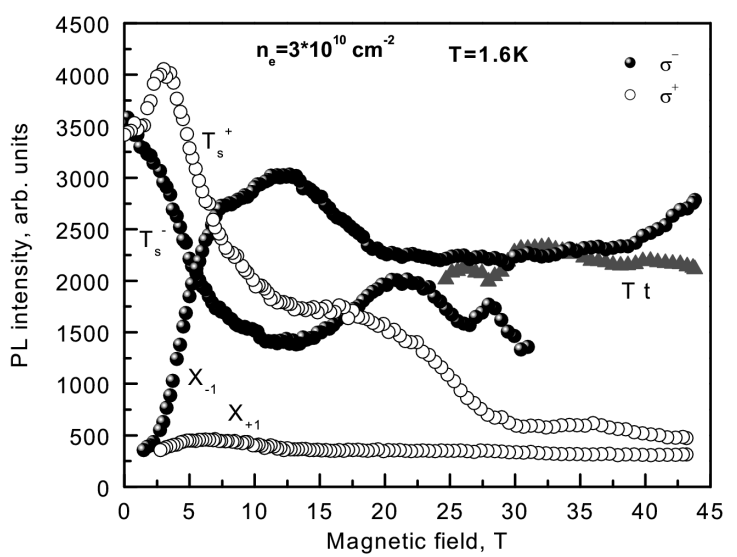

Fig. 3. Intensity of $X, T_{\mathrm{s}}$, and $T_{\mathrm{t}} \mathrm{PL}$ lines as a function of magnetic field in $\sigma^{+}$and $\sigma^{-}$polarizations. Experiment.

$\left|g_{\mathrm{e}}-g_{\mathrm{h}}\right|$. The dependence of this splitting on magnetic field is not linear. From this splitting one can determine the value of the hole $g$-factor because the electron $g$-factor is well known [11] $\left(g_{\mathrm{e}}=-1.56\right)$, and this is found to be $g_{\mathrm{h}}=+0.74$ at high magnetic fields and $g_{\mathrm{h}}=+0.2$ at small magnetic fields (see details in $[10,11]$ ).

\section{Results and discussion}

From the sign of the circular polarization of the $T_{\mathrm{t}}$ line, from its energy position and from the fact that its intensity does not depend on magnetic field, one can attribute this line to the recombination of the triplet trion with projection of the total angular momentum $(M=-1 / 2)$. The electron remaining after this recombination has spin $\left(S_{\mathrm{e}}=+1 / 2\right)$. The Zeeman splitting of this state is characterized by a total $g$-factor $\left|2 g_{\mathrm{e}}-g_{\mathrm{h}}\right|=3.8$. At the same time the observable Zeeman splitting of the triplet state should be characterized by $g$-factor $\left|g_{\mathrm{e}}-g_{\mathrm{h}}\right|$ because of the Zeeman splitting of the final state. Subtracting the value of this Zeeman splitting from the observed linear magnetic field shift of the $T_{\mathrm{t}}$ line (Fig. 4), one can determine that the binding energy of the triplet state in $\mathrm{CdTe} / \mathrm{CdMgTe} \mathrm{QWs}$ increases in magnetic field approximately linearly from 0 to $\approx 3 \mathrm{meV}$ at $50 \mathrm{~T}$ in correspondence with theoretical predictions $[6,12,5]$. This state should be optically forbidden because of conservation of angular momentum according to Table.

A model calculation using the system of kinetic equations describing the PL of the exciton-trion system was performed. Figure 4 shows the calculated intensities of all observed PL lines for a QW with electron concentration of $3 \times 10^{10} \mathrm{~cm}^{-2}$. We used the following parameters [10, 13]: exciton and singlet trion radiative lifetime $\tau_{\mathrm{Ex}}^{0}=60 \mathrm{ps}, \tau_{\mathrm{Ts}}^{0}=60 \mathrm{ps}$, electron and hole spin relaxation 


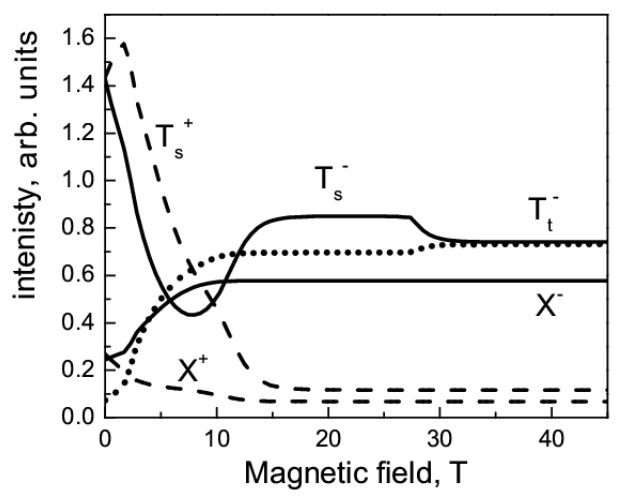

Fig. 4. Intensity of $X, T_{\mathrm{s}}$, and $T_{\mathrm{t}} \mathrm{PL}$ lines as a function of magnetic field in $\sigma^{+}$and $\sigma^{-}$polarizations. Calculation.

TABLE

Optical transitions.

\begin{tabular}{c|c|c|c|c|c|c}
\hline \hline $\begin{array}{c}\text { Projection } \\
\text { of total } \\
\text { electron } \\
\text { spin } S_{\mathrm{e}}\end{array}$ & $\begin{array}{c}\text { Projection of } \\
\text { trion spin } \\
S_{\mathrm{t}}=S_{\mathrm{e}}+S_{\mathrm{hh}}\end{array}$ & $\begin{array}{c}\text { Projection of } \\
\text { total angular } \\
\text { momentum } \\
J=S_{\mathrm{t}}+L\end{array}$ & $\begin{array}{c}\text { Absor- } \\
\text { ption }\end{array}$ & PL & $\begin{array}{c}\text { Expected } \\
\text { energy } \\
\text { position }\end{array}$ & \\
\hline \multirow{2}{*}{+1} & $+5 / 2$ & $+3 / 2$ & & & & \\
\cline { 2 - 7 } & $-1 / 2$ & $-3 / 2$ & $\sigma^{-}$ & $\sigma^{-}$ & $\left|3 g_{\mathrm{e}}-g_{\mathrm{h}}\right| / 2-E_{\mathrm{t}}$ & $T_{\mathrm{t}}^{b 2}$ \\
\hline \multirow{2}{*}{0} & $+3 / 2$ & $+1 / 2$ & $\sigma^{+}$ & $\sigma^{+}$ & $\frac{1}{2}\left|g_{\mathrm{e}}-g_{\mathrm{h}}\right|-E_{\mathrm{t}}$ & $T_{\mathrm{t}}^{b 1}$ \\
\cline { 2 - 7 } & $-3 / 2$ & $-5 / 2$ & & & & \\
\hline \multirow{2}{*}{-1} & $+1 / 2$ & $-1 / 2$ & & $\sigma^{-}$ & $-\frac{1}{2}\left|g_{\mathrm{e}}-g_{\mathrm{h}}\right|-E_{\mathrm{t}}$ & $T_{\mathrm{t}}$ \\
\cline { 2 - 7 } & $-5 / 2$ & $-7 / 2$ & & & & \\
\hline
\end{tabular}

times $\tau_{\mathrm{e}}^{\mathrm{s}}=60 \mathrm{ps}, \tau_{\mathrm{h}}^{\mathrm{s}}=30 \mathrm{ps}$ and the trion formation time $\tau_{\text {form }}=11 \mathrm{ps}$. The lifetime of the trion triplet state was taken to be $\tau_{\mathrm{Tt}}^{0}=50 \tau_{\mathrm{Ts}}^{0}$.

As is seen from Fig. 4, even though the probability of the optical transition from the triplet trion state is two orders of magnitude smaller than the probability of the allowed optical transitions, the triplet trion PL line becomes brightly observable in high magnetic fields and its intensity is comparable with the intensity of the exciton recombination line. This is due to the preferential population of exactly this triplet trion state in the magnetic field.

The upper Zeeman sublevel of the singlet trion becomes populated when the electron from the lower Zeeman sublevel (spin $-1 / 2)$ and the exciton from the upper Zeeman sublevels $(+1 / 2,-3 / 2)$ and $(+1 / 2,+3 / 2)$ become bound. It is evident that at low temperature in high enough magnetic field the concentration of such excitons is low and thus the concentration of the trions on the upper 


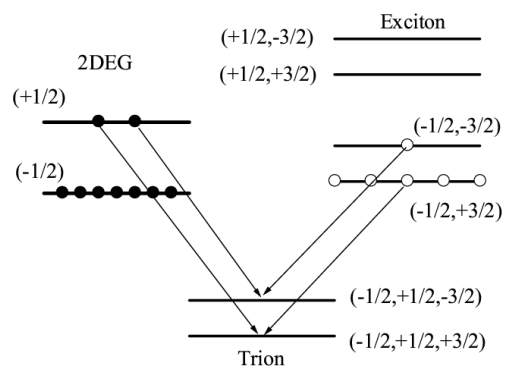

Fig. 5. Scheme of the singlet trion formation by coupling of electron and exciton.

sublevels is also low. The lower Zeeman sublevel of the singlet trion becomes populated when the electron from the upper sublevel $(+1 / 2)$ and the exciton from the lower sublevels $(-1 / 2,+3 / 2)$ and $(-1 / 2,-3 / 2)$ become bound (see Fig. 5$)$. In high magnetic field the concentration of such trions is also low due to low population of the upper electron sublevel [10, 14].

The triplet state of the trion is formed when the electron and the exciton from the lower Zeeman sublevels become bound. The population of these sublevels rises with the magnetic field and thus the concentration of this trion can appear to be rather high.

Thus when the magnetic field increases, the intensity of the luminescence line of the singlet trion state $T_{\mathrm{s}}$ should decrease in both circular polarizations and the intensity of the exciton recombination from the lower Zeeman sublevel should rise, even when the trion formation time is smaller compared to the lifetime of the exciton $\left(\tau_{\text {form }} \ll \tau_{\text {Ex }}^{0}\right)$. At the same time the intensity of the triplet line $T_{\mathrm{t}}$ can rise significantly as is seen from Fig. 4 . This growth of the intensity of triplet recombination is exactly due to the fact that the recombination channel through the singlet trion state is suppressed in the magnetic field.

This conclusion is confirmed by the temperature dependence of the PL spectra. We found that with increasing temperature the PL line of the triplet trion becomes apparent in the spectrum in higher magnetic fields. At $4.2 \mathrm{~K}$ it appeared only in $40 \mathrm{~T}$ field and at $15 \mathrm{~K}$ it was not observed even in $45 \mathrm{~T}$ field while at the same time growth of the intensity of the singlet trion recombination line was observed.

We also performed a study of reflectivity spectra of our structures. Reflectivity spectra of the structure with electron concentration of $3 \times 10^{10} \mathrm{~cm}^{-2}$ are presented in Fig. 6 in magnetic fields of 0,27 , and $45 \mathrm{~T}$ for $\sigma^{+}$and $\sigma^{-}$circular polarizations. The designation of the lines is the same as in Fig. 1. The energy positions of the reflectivity lines coincide with the energy positions in the PL spectra. In high magnetic field (Fig. 6c) a small bend near the lower Zeeman component of the reflectivity line is observed $\left(T_{\mathrm{t}}^{\mathrm{b} 1}\right)$. This line can be connected with the photocreation of the triplet state (see Table). This transition is only allowed when the spin-orbit interaction is taken into account. We have not found the state $T_{\mathrm{t}}^{\mathrm{b} 2}$ 


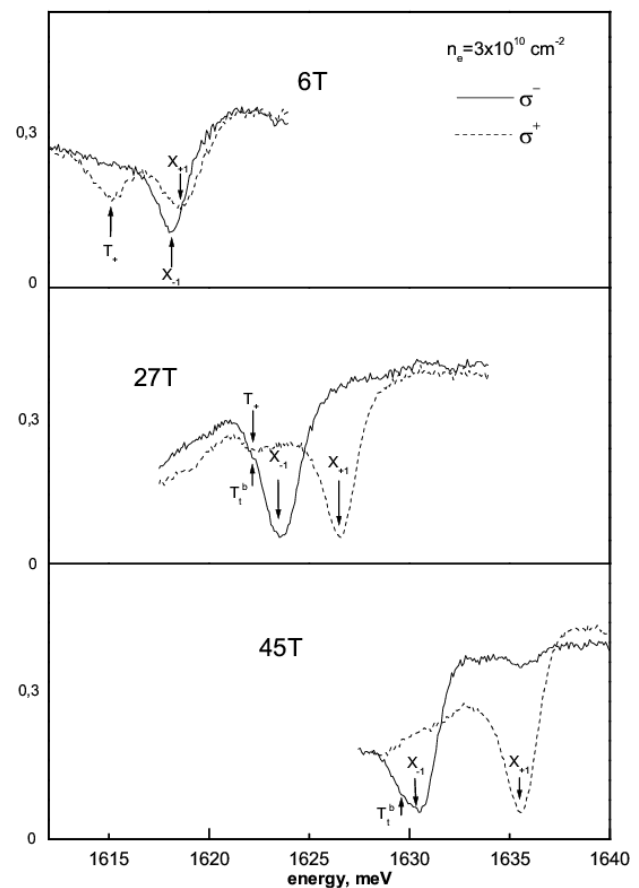

Fig. 6. Reflectivity spectra for $\mathrm{CdTe} / \mathrm{Cd}_{0.7} \mathrm{Mg}_{0.3} \mathrm{Te} \mathrm{SQW}$ structure with electron density $3 \times 10^{10} \mathrm{~cm}^{-2}$ in magnetic fields $0,27 \mathrm{~T}$, and $45 \mathrm{~T}$ at $1.6 \mathrm{~K}$ in the left $\sigma^{-}$and right $\sigma^{+}$circular polarizations. Arrows $\left(X_{-1}, T_{-}\right)\left(X_{+1}, T_{+}\right)$mark the exciton and trion lines in $\sigma^{-}$and $\sigma^{+}$polarizations.

in our spectra (see Table). In the PL spectra, this line is not observed because in the lower energy range there are other trion states into which relaxation with spin-flip processes for electron and/or hole takes place.

\section{Conclusion}

The recombination line of the triplet trion state was found in the PL spectra of modulation doped QW structures based on $\mathrm{CdTe} / \mathrm{CdMgTe}$. A model calculation of the PL spectra in the magnetic field taking into account singlet and triplet states of the trion was carried out. It is shown that the dark triplet becomes visible in the PL spectra because it becomes the only recombination channel, due to the fact that it is difficult to form a singlet state of a trion in the magnetic field. This conclusion is confirmed by the temperature dependence of the PL spectra.

\section{Acknowledgments}

The work was supported in part by grants from RFBR, the program "Nano-structures" of the Russian Ministry of Sciences and the programs of the Presidium of the RAS. 


\section{References}

[1] K. Kheng, R.T. Cox, Y. Merle d'Aubigné, F. Bassani, K. Saminadayar, S. Tatarenko, Phys. Rev. Lett. 71, 1752 (1993).

[2] O. Homburg, K. Sebald, P. Michler, J. Gutowskiet, H. Wenisch, D. Hommel, Phys. Rev. B 62, 7413 (2000).

[3] A.J. Shields, M. Pepper, M.Y. Simmons, D.A. Ritchie, Phys. Rev. B 52, 7841 (1995); G. Finkelstein, H. Shtrikman, I. Bar-Joseph, Phys. Rev. B 53, R1709 (1996).

[4] T. Vanhoucke, M. Hayne, M. Hanini, V.V. Mochalkov, Phys. Rev. B 63, 125331 (2001).

[5] C. Riva, F. Peters, K. Vagra, Phys. Rev. B 63, 115302 (2001); A.B. Dzyubenko, A.Yu. Sivachenko, Phys. Rev. Lett. 84, 4429 (2000).

[6] D. Sanvitto, D.M. Whittaker, A.J. Shields, M.Y. Simmons, D.A. Ritchie, M. Pepper, Phys. Rev. Lett. 89, 246805 (2002).

[7] D.R. Yakovlev, G.V. Astakhov, W. Ossau, S.A. Crooker, K. Uchida, N. Miura, A. Waag, N.A. Gippius, A.Yu. Sivachenko, A.B. Dzyubenko, Phys. Status Solidi B 227, 353 (2001).

[8] D.R. Yakovlev, V.P. Kochereshko, W. Ossau, J.X. Shen, A. Waag, G. Landwehr, P.C.M. Christianen, J.C. Maan, J. Cryst. Growth 184/185, 818 (1998).

[9] V.P. Kochereshko, D.R. Yakovlev, R.A. Suris, W. Ossau, G. Landwehr, T. Wojtowicz, M. Kutrowski, G. Karczewski, J. Kossut, Phys. Status Solidi A 164, 213 (1997).

[10] C.R.L.P.N. Jeukens, P.C.M. Christianen, J.C. Maan, D.R. Yakovlev, W. Ossau, V.P. Kochereshko, T. Wojtowicz, G. Karczewski, J. Kossut, Phys. Rev. B 66, 235318 (2002).

[11] A.A. Sirenko, T. Ruf, M. Cardona, D.R. Yakovlev, W. Ossau, A. Waag, G. Landwehr, Phys. Rev. B 56, 2114 (1997).

[12] D.M. Whittaker, A.J. Shields, Phys. Rev. B 56, 15185 (1997).

[13] E. Vanelle, M. Paillard, X. Marie, T. Amand, P. Gilliot, D. Brinkmann, R. Lévy, J. Cibert, S. Tatarenko, Phys. Rev. B 62, 2696 (2000).

[14] D.R. Yakovlev, V.P. Kochereshko, W. Ossau, G. Landwehr, P.C.M. Christianen, J.C. Maan, T. Wojtowicz, G. Karczewski, J. Kossut, in: Proc. ICPS-24, Jerusalem 1998, Ed. D. Gershoni, World Scientific, Singapore 1999, published on CD. 